\title{
Commentary: Engineering an optimal mechanical circulatory support system for the cavopulmonary connection
}

\author{
Osami Honjo, MD, PhD, ${ }^{a}$ Matthew G. Doyle, $\mathrm{PhD},{ }^{\mathrm{b}, \mathrm{c}}$ and Cristina H. Amon, $\mathrm{ScD}^{\mathrm{b}, \mathrm{d}}$
}

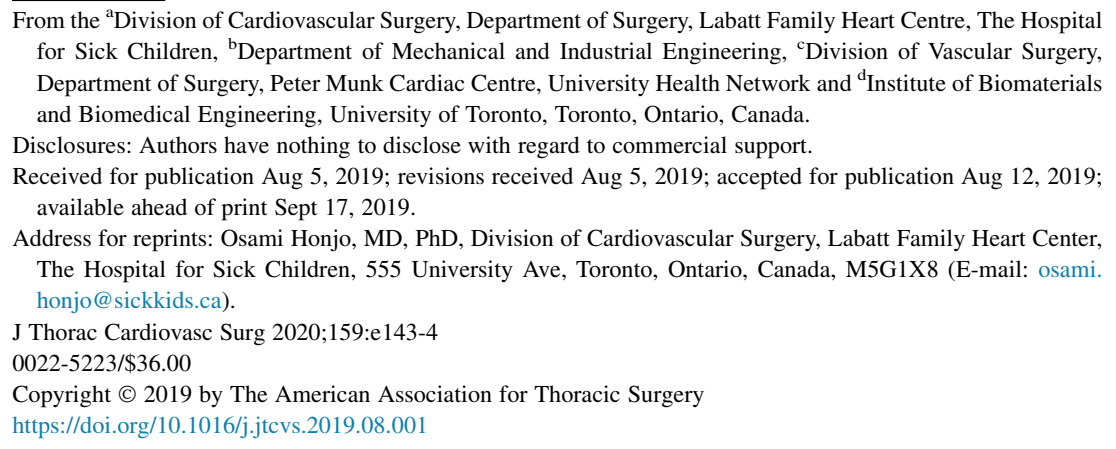

Long-term survival of the patients with a functional singleventricle physiology has dramatically improved with the establishment of staged surgical palliation and subsequent Fontan operation. As the number of the patients who live with a Fontan circulation has been increasing exponentially, management of the failing Fontan circulation becomes one of the most critical issues in pediatric and adult congenital heart disease care. Dr Trusty and colleagues ${ }^{1}$ reported an in vitro analysis of the mechanical circulatory support (MCS) system for failing Fontan circulation using a PediMag (Thoratec Corp, Pleasanton, Calif) or CentriMag (Thoratec Corp) centrifugal pump. This group pioneered the computational and experimental flow dynamics of the single-ventricle and cavopulmonary physiology. The authors and the group should be congratulated on their significant contributions to the field over the years and on the new knowledge delivered in this article.

The configuration of this particular MCS system is to drain from the inferior vena cava and to pump into the central pulmonary artery, that is, the intersection between the systemic venous cannels and the branch pulmonary arteries, through cannulae attached to the centrifugal pump. The authors used a small pump (PediMag) and a large pump (CentriMag) in a mock flow loop to test the efficacy of the MCS system based on the pump size and the flow rate. One of the most significant challenges of supporting the Fontan circulation is its nonvalve nature of the Fontan pathway. Unlike a standard ventricular assist device in which a competent aortic valve between the inflow and outflow cannulae prevents significant recirculation, any proposed MCS system for the failing Fontan circulation needs to overcome the recirculation issue. Banding the cavopulmonary pathway is a common method to theoretically overcome the issue, ${ }^{1,2}$ although in the clinical setting,

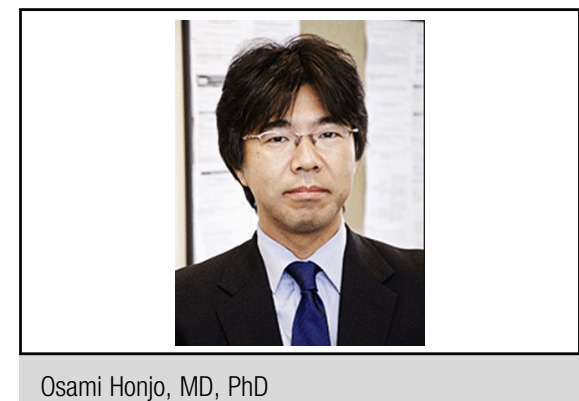

Osami Honjo, MD, PhD

Central Message

Dr Trusty and colleagues reported a novel mock loop experiment of MCS for failing Fontan circulation.

See Article page 1413 in the November 2019 issue.

banding the Fontan pathway is not realistic because of the thrombosis risk. There is a successful clinical case of MCS for the failing Fontan circulation using a Berlin Heart EXCOR (Berlin, Germany), which proves the concept of a "valved" MCS effective supporting the cavopulmonary physiology. ${ }^{3}$ One of the most interesting points from this study is that the large CentriMag pump achieved effective cavopulmonary support, confirmed by lowering pressure in the inferior vena cava and increasing pulmonary artery pressure effectively while maintaining a cardiac output of $5 \mathrm{~L} / \mathrm{min}$. What is interesting is that dye flow visualization showed the blood flow from the superior vena cava was pulled down into the inflow cannula while the effective pulmonary artery forward flow was maintained without recirculation. Although we cannot fully explain why the pulmonary blood flow was not pulled down while the superior vena cava blood flow was pulled to the pump, the data, combined with the mock loop flow dye flow visualization, are convincing.

When designing an optimal MCS system for the failing Fontan circulation, one of the most important factors to consider is its implantation design. Because patients with a failing Fontan circulation tend to be clinically ill and have multiple previous open surgeries, MCS implantation as an open procedure is less attractive. Therefore, percutaneous insertion of such system is drawing much attention. The MCS system developed by Dr Rodefeld and 
colleagues, ${ }^{4}$ another pioneering group in the field, is a great example of such a system that potentially can be inserted percutaneously. Our group recently published a new design of a percutaneously implantable MCS device for this population. ${ }^{5}$ Our design is also remarkable for its outflow tip design to prevent recirculation at the "intersection."

Several groups have been working on the development of an optimal MCS system for this particular failing circulation for decades, and yet we have not come up with a system that is clinically applicable. This study, however, certainly adds substantial new knowledge to advance this field.

\section{References}

1. Trusty PM, Tree M, Maher K, Slesnick TC, Kanter KR, Yoganathan AP, et al. An in vitro analysis of the PediMag and CentriMag for right-sided failing Fontan support. J Thorac Cardiovasc Surg. 2019;158:1413-21.

2. Zhu J, Kato H, Fu YY, Zhao L, Foreman C, Davey L, et al. Cavopulmonary support with a micro-axial pump for the failing Fontan physiology. ASAIO J. 2015;61: 49-54.

3. Prêtre R, Häussler A, Bettex D, Genoni M. Right-sided univentricular cardiac assistance in a failing Fontan circulation. Ann Thorac Surg. 2008;86: 1018-20.

4. Rodefeld MD, Frankel SH, Giridharan GA. Cavopulmonary assist:(Em) powering the univentricular Fontan circulation. Semin Thorac Cardiovasc Surg Pediatr Card Surg Annu. 2011;14:45-54.

5. Lin WCP, Doyle MG, Roche SL, Honjo O, Forbes TL, Amon CH. Computational fluid dynamic simulations of a cavopulmonary assist device for failing Fontan circulation. J Thorac Cardiovasc Surg. 2019;158:1424-33.e5. 